Revue d'histoire de l'Amérique française

REVUE D.HISTOIRE DE L'AMÉRIQUE FRANÇAISE

\title{
La censure en temps de guerre : Radio-Canada et le plébiscite de 1942
}

\section{Alain Canuel}

Volume 52, numéro 2, automne 1998

URI : https://id.erudit.org/iderudit/005347ar

DOI : https://doi.org/10.7202/005347ar

Aller au sommaire du numéro

\section{Éditeur(s)}

Institut d'histoire de l'Amérique française

ISSN

0035-2357 (imprimé)

1492-1383 (numérique)

Découvrir la revue

\section{Citer cet article}

Canuel, A. (1998). La censure en temps de guerre : Radio-Canada et le plébiscite de 1942. Revue d'histoire de l'Amérique française, 52(2), 217-242.

https://doi.org/10.7202/005347ar
Résumé de l'article

RÉSUMÉ

La présente étude se propose d'analyser, dans le contexte de la censure pendant la Deuxième Guerre mondiale, la controverse que soulève la Société Radio-Canada (SRC) lors de la campagne du plébiscite de 1942 en permettant aux tenants du OUI de se faire entendre et en refusant aux tenants du NON de présenter leur point de vue sur les ondes nationales. En plus d'exacerber les tenants du NON, cette décision mine la crédibilité de la SRC qui devient rapidement identifiée à l'appareil répressif de l'État. L'analyse remet également en question l'affirmation selon laquelle la prise de décision de la SRC lors du plébiscite aurait eu pour effet de favoriser le développement du réseau français au Canada. 


\title{
LA CENSURE EN TEMPS DE GUERRE : RADIO-CANADA ET LE PLÉBISCITE DE 1942
}

\author{
ALAIN CANUEL \\ Conseil de recherches en sciences naturelles et \\ génie du Canada
}

\section{RÉSUMÉ}

La présente étude se propose d'analyser, dans le contexte de la censure pendant la Deuxième Guerre mondiale, la controverse que soulève la Société Radio-Canada (SRC) lors de la campagne du plébiscite de 1942 en permettant aux tenants du OUI de se faire entendre et en refusant aux tenants du NON de présenter leur point de vue sur les ondes nationales. En plus d'exacerber les tenants du NON, cette décision mine la crédibilité de la SRC qui devient rapidement identifiée à l'appareil répressif de l'État. L'analyse remet également en question l'affirmation selon laquelle la prise de décision de la SRC lors du plébiscite aurait eu pour effet de favoriser le développement du réseau français au Canada.

\section{ABSTRACT}

This study proposes to analyse, in the context of censorship during the Second World War, the controversy that the Canadian Broadcasting Corporation (CBC) raised during the plebiscit campaign of 1942 in authorizing the YES advocates and in denying the NO advocates to present their point of view on the national airwaves. This decision not only exacerbates the $N O$ advocates but also undermines the CBC's credibility which rapidly becomes identified as the repressive apparatus of the State. The analysis also questions the assertion which tends to prove that it was the CBC's decision during the plebiscit that would have favoured the development of the French network in Canada.

\section{INTRODUCTION}

Au cours de la seconde moitié du XIX ${ }^{\mathrm{e}}$ siècle, les technologies de communication telles le câble sous-marin et la télégraphie n'ont cessé d'accroître la puissance et le prestige des nations qui ont été les premières à les utiliser et à les exploiter à l'échelle nationale et internationale. Ces moyens de communication sont rapidement devenus de puissants leviers politiques et militaires pour la conquête du monde et 
le contrôle des colonies et dépendances ${ }^{1}$. Au début du $\mathrm{XX}^{\mathrm{e}}$ siècle, la radio, qui s'inscrit dans le sillage de ces technologies, fait appel à une autre dimension de la communication: à la différence du câble sousmarin et de la télégraphie qui privilégient une communication de personne à personne, la radio rejoint un auditoire de masse. Son message devient facilement accessible à des milliers, voire à des millions d'auditeurs, en particulier lorsque les stations radiophoniques forment un réseau pour diffuser leur programmation à l'échelle nationale. En outre, le caractère social de la radio favorise une communication des idées et des sentiments qui, le plus souvent, séduit ou influence les auditeurs. Mais il y a l'envers de cette réalité: en temps de guerre, la radio peut se transformer en une arme redoutable. «Nul n'ignore, d'affirmer Paul Legendre, que dans les pays totalitaires [la radio] a servi et sert encore à l'oppression systématique de la pensée sociale et politique.» Et l'auteur de poursuivre: «Qui dira le rôle qu'elle a joué dans la conscience du peuple allemand ${ }^{2} \ldots »$

Qu'en est-il de la radio au Canada pendant la Deuxième Guerre mondiale? Comment et à quelles fins est-elle utilisée? Peut-on parler de propagande dont les méthodes et l'efficacité visent à manipuler l'opinion publique? Peut-on imaginer que l'information soit dirigée ou qu'il y ait désinformation? Le décret qui établit la censure des médias en temps de guerre (6 septembre 1939) se fonde sur une politique de bonne volonté et de coopération de la presse. Dans l'esprit du gouvernement canadien, cette politique doit «permettre la libre publication des nouvelles et l'expression d'opinions pour autant que ces dernières soient conciliables avec la protection des secrets militaires et qu'elles n'affectent pas le moral de guerre au Canada ${ }^{3}{ }$. Toutefois, protéger le moral des militaires et de la population se traduit, selon Claude Beauregard, par une censure politique qui limite la possibilité de critiquer l'action gouvernementale et militaire. Cette censure, poursuit-il, est subtile dans son application, car la protection du moral demande à être interprétée et

1. Voir particulièrement Charles Lesage, La rivalité anglo-germanique. Les câbles sousmarins allemands (Paris, 1915); R. Hennig, «Die deutsche Seekabelpolitik zur Befreiung von englischen Weltmonopol», Meereskunde, 6 (1912): 1-34; P. Lertes, Die drahtlose Telegraphie und Telephonie (Dresden, Leipzig, 1922); Alain Canuel, «Le câble sous-marin et la TSF en Allemagne avant la Première Guerre mondiale», Annales canadiennes d'histoire, 26,3 (décembre 1991): 415429 .

2. Paul Legendre, La radio, puissance sociale (Ottawa, Institut littéraire du Québec, 1951), $10-11$.

3. Archives nationales du Canada (ci-après ANC), RG2, Série 14, vol. 5968, dossier 1-G/ 90, Report Upon Activities of Press Censorship, $1^{\text {er }}$ septembre 1939 au 31 mars 1940, et particulièrement le sous-titre «Press Censorship Policy». 
devient sujette à des considérations beaucoup plus politiques que juridiques. Il y a déviance de la sphère juridique ou légale (la loi qui autorise la censure) vers la sphère politique ${ }^{4}$. Dès lors, la censure à caractère politique occupe un champ d'intervention aux formes multiples et peut même s'associer à la propagande dans l'intérêt de la défense nationale ou de la sécurité publique ${ }^{5}$.

Au début des années 1940, la coopération entre les censeurs et la presse ne semble pas donner satisfaction: «La censure de la presse fonctionne de moins en moins bien et les journaux ne tiennent pas compte des mesures positives ${ }^{6} . »$ Le 6 mars 1941, le Premier ministre, William Lyon Mackenzie King, fait parvenir à tous les journaux canadiens une lettre accompagnée d'un Mémoire concernant les dangers résultant de la publication de certaines nouvelles dans la presse canadienne et les invite à suivre scrupuleusement les consignes des censeurs. La conduite latitudinaire de certains journaux ne fait qu'aggraver la situation, au point de discréditer le pouvoir des censeurs qui se voient désormais contraints d'engager des poursuites. Il ne faudrait pas croire cependant que ces actions juridiques vont modifier de façon radicale les comportements de la presse. Dans les faits, les journalistes continuent de publier sans trop se soucier des règlements de la censure, sachant pertinemment qu'il n'y a aucun texte de loi qui définit les fonctions des censeurs, du moins jusqu'en août 1942. En revanche, le gouvernement King, pour faire bonne figure devant la population canadienne, interdit toute propagande subversive et se dit prêt à dénoncer tout discours tendancieux qui pourrait être exercé sur l'opinion publique ou qui serait susceptible de mettre en danger le principe même de la démocratie canadienne ${ }^{7}$. Malgré la mise en place d'une censure appuyée par un imposant arsenal de règlements et les déclarations à l'emporte-pièce de députés et ministres fédéraux, le véritable cheval de bataille du gouvernement King, lors du conflit mondial, n'est pas tant l'application des

4. Claude Beauregard, Guerre et censure: l'expérience des journaux, des militaires et de la population pendant la Deuxième Guerre mondiale, thèse présentée à la Faculté des études supérieures de l'Université Laval, mars 1995, 7-8.

5. ANC, RG2, Série 14, op. cit., au sous-titre «Subversive Propaganda»; voir également, ANC, RG2, Série 14, vol. 5942, dossier 1, Comité de coordination de la censure. Manuel concernant la censure de la presse et de la radiodiffusion (Ottawa, J.-O. Patenaude, mars 1940).

6. Claude Beauregard, Guerre et censure..., 93.

7. À propos de la propagande subversive, voir: Canada, Chambre des communes, Débats (ci-après Débats), 22 mars 1939: 2181; 22 mai 1940: 118; 27 mai 1940: 223; Canada, House of Commons, Special Committee on Radio Broadcasting (ci-après SCRB), Minutes of Proceedings and Evidence (Ottawa, F. A. Acland, 1942), 89, 172, 264. 
règles de la censure et la dénonciation de la propagande subversive que la crise de la conscription qui risque de compromettre l'unité nationale ${ }^{8}$.

Le 27 avril 1942, le Premier ministre entend recourir au plébiscite pour demander à la population canadienne si elle consent «à libérer le gouvernement de toute obligation résultant d'engagements antérieurs qui restreignent les méthodes de recrutement pour le service militaire ${ }^{9} \gg$. En vue de la campagne d'information sur le plébiscite, la Société RadioCanada (SRC) décide de mettre à la disposition des chefs de partis politiques - essentiellement les partis libéral et conservateur, le Crédit social et la Cooperative Commonwealth Federation - une durée égale de temps d'antenne afin qu'ils puissent faire connaitre leur point de vue à la population. Cette décision donne prise à la critique et soulève le mécontentement d'une partie de la population canadienne puisqu'elle permet uniquement à certains porte-parole politiques de faire connaître leur point de vue. En excluant les partisans du NON, par l'application d'une politique qui définit les règles en période de campagne électorale fédérale, la SRC suscite la controverse, ce qui lui porte préjudice. D'aucuns dénoncent alors la société d'État comme une «institution qui [dépend] d'Ottawa et qui se [comporte] comme une officine de propagande gouvernementale ${ }^{10}$.

Notre étude se propose d'analyser la controverse que soulève la SRC lors du plébiscite de 1942 par l'application de règles «électorales» et ses effets, à court et à moyen termes: d'une part, l'exacerbation des discours de certains individus ou groupes qui estiment avoir été lésés dans leurs droits et liberté d'expression; d'autre part, la perte de crédibilité de la société d'État. Nous commenterons enfin le lien de causalité que certains auteurs établissent entre le plébiscite de 1942 et l'évolution du réseau français de Radio-Canada.

8. «La propagande de guerre, au plan national, a essentiellement pour objectif de concilier deux nationalismes, québécois/canado-anglophone, qui s'affrontent et nuisent à l'unité de la conscience nationale et de l'effort de guerre», dans Renée Legris, Propagande de guerre et nationalisme dans le radio-feuilleton (1939-1959) (Montréal, Fides, 1981), 21. Pour le Premier ministre, l'unité nationale représente un but, non un moyen. Voir J. L. Granatstein, Conscription in the Second World War, 1939-1945. A Study of Political Management (Toronto, Ryerson Press, 1969), 13, 74; Jean-Yves Gravel, Le Québec et la guerre (Montréal, Boréal Express, coll. «Études d'histoire du Québec», n 7, 1974), 77 et ss.

9. Débats (11 mai 1942), 2354; La Ligue pour la défense du Canada: ce qu'elle a fait, son attitude présente, ce qu'elle fera (Montréal, Imprimerie populaire, s.d.), 3.

10. André Laurendeau, La crise de la conscription 1942 (Montréal, Éditions du Jour, 1962), 103. Le député du Bloc populaire, Maxime Raymond (circonscription de Beauharnois-Laprairie) qualifie la SRC de «succursale politique du gouvernement», dans Débats (4 juillet 1944), 4687. 


\section{1 - LA POLITIQUE DE LA SOCIÉTÉ RADIO-CANADA}

Dans un document daté du 8 juillet 1939, le Bureau des gouverneurs de Radio-Canada élabore les lignes directrices de la politique que doit suivre la société d'État en période de campagne électorale fédérale. Il est stipulé, entre autres, que la SRC doit mettre à la disposition des partis politiques une période égale et gratuite de temps d'antenne pour s'adresser à la population et qu'un tel arrangement ne peut être conclu qu'avec les partis politiques et non avec les individus, quelle que soit leur notoriété. Les gouverneurs prennent également les dispositions nécessaires afin que les auditeurs puissent bénéficier du point de vue de tous les partis politiques existants. Selon eux, la société d'État ne doit pas être limitative dans le sens qu'elle ne doit pas favoriser uniquement la représentation des partis politiques reconnus à la Chambre des communes. Une telle limitation, affirme-t-on, serait injuste à l'endroit des nouveaux partis qui représentent, à l'échelle nationale, une part importante de l'opinion publique du pays. Enfin, la SRC affirme qu'elle entend «résister à tout effort tenté dans le but d'enrégimenter l'opinion publique ou d'étouffer la liberté de parole. Nous croyons que la société d'État, soutiennent les gouverneurs, doit favoriser autant que faire se peut l'utilisation des ondes pour stimuler une franche discussion sur toute question sujette à controverse ${ }^{11}$.»

L'ensemble des dispositions contenues dans le document rédigé par le Bureau des gouverneurs permet d'assurer l'équité entre les partis politiques, d'informer, selon divers points de vue, la population canadienne sur des questions d'intérêt national et de garantir en tout temps l'impartialité de la société d'État. Initialement, ce document, qui a subi un premier test lors des élections fédérales de 1940, a été accueilli favorablement dans les milieux concernés. Cela ne surprend guère puisque le Bureau des gouverneurs s'est inspiré des principes généraux de la radiodiffusion britannique en période électorale, tout en introduisant quelques modifications pour tenir compte de la réalité canadienne. Cependant, l'application des mêmes règles pour la tenue d'un plébiscite pose, comme nous l'avons mentionné précédemment, un problème majeur puisqu'elle exclut d'emblée toute représentation du NON.

11. Archives de l'Université Laval (ci-après AUL), Fonds Adrien-Pouliot, P168/3/1/4/2, «A Statement of Policy of the CBC to Controversial Broadcasting». Issued by the Authorithy of the Board of Governors, July 8, 1939: 1, 2, 5, 7, 14 (notre traduction). Voir également, ANC, Fonds Diefenbaker, Document M-7417, vol. 5, [White Paper on] «Political and Controversial Broadcasting Policies and Ruling». Issued by the Board of Governors, February 21, 1944: 3688-3704. 


\section{2 - LA CORRESPONDANCE LAURENDEAU-FRIGON}

La Ligue pour la défense du Canada - «née du divorce entre l'opinion officielle et la réaction populaire au Canada français» — entend faire valoir le point de vue des partisans du NON. Elle demande officiellement à Augustin Frigon, directeur général adjoint de la SRC, d'obtenir le même nombre d'heures d'antenne que celui accordé aux partisans du OUI ${ }^{12}$. L'échange de correspondance entre André Laurendeau, secrétaire de la Ligue, et Augustin Frigon, qui s'échelonne du 13 février au 11 mars 1942, sera publié dès mars 1942 presque intégralement dans Le Devoir. En plus de susciter l'intérêt des lecteurs à propos des arguments de la Ligue et de ceux de la SRC, la publication de cette correspondance amènera des commentaires critiques, parfois virulents à l'endroit de la Société RadioCanada.

La première lettre adressée à Augustin Frigon s'appuie, selon le secrétaire de la Ligue, sur le droit de tout citoyen «de répondre au plébiscite, selon son jugement et sa conscience, sans se faire taxer pour cela d'anticonscriptionniste ou de dangeureuse agitation ${ }^{13}{ }$. À cet égard, le titre et le sous-titre de l'article publié dans Le Devoir du 21 mars 1942 soulignent clairement l'enjeu du débat qui s'engage entre la Ligue et la société d'État: «Radio-Canada, le plébiscite et la Ligue pour la défense du Canada. La ligue aura-t-elle le droit de se servir de Radio-Canada? — Les "OUI" auront-ils l'exclusivité des postes nationaux à titre gratuit? Le règlement qu'invoque Radio-Canada ne s'applique pas à la situation ${ }^{14}$.» La question soulevée par André Laurendeau dans sa première lettre à Radio-Canada repose d'abord sur le droit à un traitement équitable: «Estce l'intention de la Société Radio-Canada d'accorder une chance égale, c'est-à-dire un nombre égal d'heures aux citoyens canadiens estimant que la réponse au plébiscite doit être NON, et qui voudront orienter l'opinion en ce sens ${ }^{15}$ ?»

La réponse de Frigon ne tarde pas. S'appuyant sur le principe de neutralité de la société d'État, il écrit: «Il est fort probable que nos règlements qui s'appliquent en temps d'élections générales seront mis en vigueur au cours de la campagne qui [...] précédera le plébiscite [...]. Nos règlements

12. André Laurendeau, La crise de la conscription..., 81; Centre de recherche Lionel-Groulx (ci-après CRLG), Fonds Maxime-Raymond, P3/F, 366, Lettre d'André Laurendeau à Augustin Frigon, 18 février 1942.

13. André Laurendeau, La crise de la conscription..., 84.

14. «Radio-Canada, le plébiscite et la Ligue pour la défense du Canada», Le Devoir, 21 mars 1942.

15. CRLG, Fonds Maxime-Raymond, loc. cit., 366, «Lettre d'André Laurendeau à Augustin Frigon», 13 février 1942. 
généraux ne nous permettent pas de louer nos postes à des individus ou à des groupes qui désirent traiter à la radio des questions politiques ou sujettes à controverse ${ }^{16}$.»

Laurendeau est déterminé à poursuivre son plaidoyer pour appuyer la cause du NON. Il refuse l'argument de Frigon qui assimile le plébiscite à une campagne électorale et le soumet à des règles identiques. Laurendeau établit, au contraire, une nette distinction entre ces deux réalités politiques et il met en relief le principe d'équité qui doit prévaloir lors d'un plébiscite:

Dans un plébiscite, la question est toute différente. Il ne s'agit pas alors de porter tel ou tel parti au pouvoir, mais de connaître l'opinion du peuple canadien sur telle ou telle question [...]. La radio d'État a donc une ligne de conduite toute tracée: éclairer le peuple sur les raisons qui militent en faveur du OUI et sur celles qui militent en faveur du $\mathrm{NON}^{17}$.

L'argumentation de Laurendeau qui s'appuie sur le principe de la démocratie, où prédomine le respect de la liberté et de l'égalité, ne peut qu'inciter Frigon à faire preuve d'une plus grande souplesse dans ses propos: «Étant donné l'importance du plébiscite et les conditions particulières de la campagne, qui se fera sur cette question, il est fort possible qu'une interprétation spéciale de nos règlements soit donnée par notre administration. Toute décision qui pourrait être prise à ce sujet vous sera transmise en temps et lieu ${ }^{18}$.» Dans son ouvrage intitulé La crise de la conscription, André Laurendeau commente la réponse de Frigon en affirmant que même si ce dernier maintenait sa position, on le sentait ébranlé $^{19}$. La justesse de ses propos se reflète dans une note de service que Frigon fait parvenir, le 22 février 1942, au directeur général de la SRC, Gladstone Murray:

Étant donné les conditions actuelles au Québec, je crois que nous ne pouvons permettre au présent gouvernement et autres partis de faire valoir un côté de la question et refuser aux autres de faire valoir l'autre côté de la question. Quoique nous fassions, nous devons

16. CRLG, Fonds Maxime-Raymond, loc. cit., 366, «Lettre d'Augustin Frigon à André Laurendeau», 16 février 1942.

17. CRLG, Fonds Maxime-Raymond, loc. cit., «Lettre d'André Laurendeau à Augustin Frigon», 18 février 1942.

18. CRLG, Fonds Maxime-Raymond, loc. cit., «Lettre d'Augustin Frigon à André Laurendeau», 24 février 1942.

19. André Laurendeau, La crise de la conscription..., 106. 
trouver un moyen pour que les partisans du NON puissent être entendus sur nos réseaux, du moins dans la province de Québec ${ }^{20}$.

En réalité, la souplesse dont fait preuve Frigon ne changera rien à la décision: les représentants du NON ne seront jamais autorisés à faire valoir leur point de vue sur les ondes de Radio-Canada. Néanmoins, ce changement d'attitude traduit un malaise certain chez Frigon, à la fois membre de la haute direction de Radio-Canada et représentant du réseau français au Canada. Cependant, sans mettre en cause la sincérité de Frigon, il est plus que probable que la société d'État cherchait à gagner du temps en restant dans l'expectative. Cette hypothèse repose sur le retard indu à rendre une décision définitive sur cette question. «Pendant plusieurs jours, affirme Laurendeau, la société ne bouge pas. Frigon, par téléphone, nous demande d'attendre le vote de la loi. Elle est votée. Quatre jours plus tard (9 mars), nous écrivons et demandons quelle est la décision définitive de Radio-Canada. Réponse du 11 mars: "Rien n'est encore décidé", il faut attendre encore ${ }^{21} . »$ Le directeur du Devoir, Georges Pelletier, maintient que

la Société a eu le temps de prendre une décision finale. Depuis cinq semaines, les choses restent de sa part au même point: dans l'indécision. Voudrait-on noyer le poisson — en l'espèce la demande de la Ligue - on ne manœuvrerait guère autrement [...]. Aurait-on pensé à en user comme un faux-fuyant, devant une situation embarrassante, soit pour la contourner, soit pour gagner du temps ${ }^{22}$ ?

La Ligue devra patienter jusqu'au 8 avril, soit moins d'un mois avant la tenue du plébiscite, pour apprendre que sa demande était refusée par la SRC. Dans cette affaire, peut-on croire que la SRC a maintenu son indépendance vis-à-vis du gouvernement?

\section{3 - LA LIGUE ET L'INGÉRENCE POLITIQUE À RADIO-CANADA}

Conformément aux dispositions de la Loi sur la radiodiffusion, la SRC est devenue, en 1936, l'organisme responsable de l'exploitation et de l'administration de la radio nationale au Canada. Ce mandat devait lui permettre, entre autres, de demeurer à l'abri de toute ingérence politique. Dans ce contexte, la SRC avait rendu public, le 8 juillet 1939, un

20. ANC, Fonds Mackenzie-King, MG26J1, vol. 330, «Lettre d'Augustin Frigon à Gladstone Murray», 24 février 1942: 262093.

21. André Laurendeau, La crise de la conscription..., 106; «Publication des lettres en dates des 9 et 11 mars 1942», Le Devoir, 21 mars 1942.

22. Georges Pelletier, «De qui se moquerait-on?», Le Devoir, 24 mars 1942. 
document sur la conduite qu'elle entendait adopter face à des questions ou thèmes controversés. En énonçant clairement les règles concernant l'utilisation de la radio publique en période électorale, la société d'État avait voulu se prémunir contre toute accusation de favoritisme auprès de groupes, individus ou partis politiques qui aurait pu ternir son image.

En 1942, lors du plébiscite, la question se pose différemment. L'objet de débat entre la Ligue et la SRC ne porte pas tant sur les règles elles-mêmes que sur leur application qui, d'emblée, écarte le discours du NON sur les ondes de la radio nationale. Qui plus est, cette attitude de la SRC, soutient la Ligue, est dictée par le gouvernement King. À cet égard, la lettre que le secrétariat de la Ligue adresse au ministre des Services nationaux de guerre, J. T. Thomson, fait état de certaines contradictions:

Qui faut-il croire? Que faut-il croire? Si M. King disait la vérité en novembre 1940 et si Radio-Canada est vraiment indépendante de la politique, comment M. Pouliot [membre du Bureau des gouverneurs] pouvait-il prétendre, le 14 avril 1942, que le gouvernement fédéral peut toujours intervenir «lorsqu'il le juge à propos» pour dicter une ligne de conduite à Radio-Canada, et cela sur une question aussi éminemment politique et controversée qu'un plébiscite proposé au peuple par le gouvernement, et dans lequel le gouvernement prend lui-même parti ${ }^{23}$ ?

Les déclarations contradictoires de King, de Pouliot et de Frigon ne font que renforcer la défense du NON qui se cristallise autour de l'ingérence politique ${ }^{24}$. La partialité du gouvernement dans cette affaire a vite fait d'inciter les partisans du NON à mettre en œuvre une stratégie de coopération. Désormais, des groupes provinciaux et régionaux (Union catholique des cultivateurs, Association professionnelle des voyageurs de commerce, Association catholique de la jeunesse canadienne-française, Association canadienne-française d'éducation d'Ontario, Jeunesse étudiante catholique, Jeunesse ouvrière catholique, etc.), des 1942.

23. André Laurendeau, «Veut-on miner le prestige de Radio- Canada?»", Le Devoir, 21 avril

24. La thèse de l'ingérence politique n'est pas nouvelle. En 1939, lorsque le Canada déclare officiellement la guerre à l'Allemagne, le ministre C. D. Howe entend remplacer le Bureau des gouverneurs par un comité exécutif relevant directement de lui. L'opposition conservatrice réclamera à maintes reprises une enquête parlementaire pour redéfinir les responsabilités hiérarchiques de la SRC. Voir David Ellis, La radiodiffusion canadienne: objectifs et réalités, 1928-1968 (Hull, Centre d'édition du gouvernement du Canada, 1979), 26. L'Association des radiodiffuseurs canadiens dénonce aussi cette ingérence en déclarant: «le gouvernement (c'est-à-dire le Premier ministre et son cabinet) contrôle la SRC», dans ANC, Fonds Diefenbaker, loc. cit., 1940-1956, Series, vol. 7: 5162 . 
syndicats catholiques, des corporations municipales et d'autres organismes reconnus (Ligue d'Action nationale et Société Saint-Jean-Baptiste) appuient ouvertement la Ligue.

Parmi tous ces appuis, la Société Saint-Jean-Baptiste (SSJB) demeure l'organisation la plus influente et la mieux structurée pour intervenir efficacement dans ce débat, pour développer une série d'actions concertées à travers le Canada et pour exercer des pressions auprès du gouvernement de l'heure. Dès octobre 1941, la SSJB de Montréal faisait connaître aux médias et au gouvernement King sa position sur la politique de guerre, voulant ainsi prévenir toute manœuvre militaire ou politique qui pourrait nuire aux intérêts de ses membres. Dans une lettre adressée au Premier ministre canadien, la société énumérait les résolutions qu'elle avait adoptées en réunion plénière, à savoir:

- la continuation de l'appui du Canada aux pays alliés;

- la limitation des dépenses de guerre aux ressources du pays et appliquées en premier lieu à la défense du Canada;

- l'opposition indéfectible à toute mesure de conscription, ouverte ou déguisée, pour service outre-mer ${ }^{25}$.

Le 22 janvier 1942, lorsque le gouvernement King annonce dans son Discours du Trône la tenue d'un plébiscite sur la conscription, la SSJB met en branle «une campagne d'opinion publique pour engager la population à voter: non». Il n'y a pas de doute que cette action relève du mandat des sociétés Saint-Jean-Baptiste, selon Édouard Coulombe, président de la SSJB du diocèce de Québec ${ }^{26}$. Pour sa part, dans une lettre adressée au secrétaire de la Ligue, le président de la SSJB, section l'Assomption, écrit: «Encouragé par des hommes comme vous, le Comité d'Action Nationale est décidé à marcher de l'avant et veut éclairer la population du Comté de l'Assomption [...]. Aussi, avons-nous décidé de faire nous aussi notre part et de former un comité local de la Ligue pour la défense du Canada ${ }^{27}$.» Face à l'expectative de RadioCanada vis-à-vis des tenants du NON, la SSJB appuie la Ligue en exerçant des pressions sur le directeur de la société d'État, Gladstone Murray, pour qu'il y ait «libre expression des opinions honnêtes et des idées

25. Archives nationales du Québec (ci-après ANQ, Fonds SSJB), Fonds Société Saint-JeanBaptiste de Montréal, 06-M, P82/124-1888, document microfilmé no 9157: 2478-2479.

26. Édouard Coulombe, «Les sociétés Saint-Jean-Baptiste en face du plébiscite», L'Action nationale, 19 (1942): 206-211, citation, 208.

27. CRLG, Fonds Ligue pour la Défense du Canada, P6/ A, 4, «Lettre de F. Coiteux à André Laurendeau», 23 février 1942. Il ne s'agit-là qu'une des nombreuses lettres d'appui à la Ligue qui proviennent de différentes régions du Québec. 
justes sur des questions et des problèmes d'ordre public et national ${ }^{28}$ ». Considérée comme le maillon le plus important à l'échelle du pays, la SSJB de Montréal utilise également son réseau de liaison postale pour faire connaître sa position aux autres sections canadiennes afin qu'elles puissent mener «une campagne de renseignement [visant] à produire de l'action défensive». Le message atteint rapidement son but: «Le 5 février dernier vous nous écriviez nous annonçant la fondation du Comité de Défense du Canada et nous envoyant aussi une directive relativement au plébiscite. [...] Nous croyons que ce mode de transmettre les résultats de vos délibérations est très avantageux pour nous et nous espérons que vous voudrez bien continuer à nous suggérer des directives ${ }^{29}$.»

De son côté, la Ligue organise, en collaboration avec des groupes régionaux, de nombreuses assemblées anticonscriptionnistes à Montréal et ailleurs au Québec. La multiplication des activités du NON engendrées par le refus de Radio-Canada ne suffit toutefois pas à expliquer les résultats du plébiscite. À l'instar des autres provinces, le Québec a voté sur une question de fond, cela s'entend. Nous pouvons tout de même nous interroger sur le sentiment de frustration général qu'éprouvent les tenants du NON face à Radio-Canada. Ceux qui comptaient utiliser la radio nationale pour faire valoir leur opinion ont vu leurs espoirs s'évanouir quelques semaines avant la consultation fédérale. La seule alternative qui s'offre alors à la Ligue est de louer du temps d'antenne aux stations privées. Mais cela coûte cher et la Ligue n'a pas les fonds nécessaires pour atteindre, avec le même degré d'efficacité que le réseau national, la population canadienne. Quelques émissions anticonscriptionnistes et quelques déclarations officielles à la radio privée ne suffisent pas à galvaniser les auditeurs. Sur le plan technique, la campagne pour le NON s'organise péniblement. Par exemple, un discours enregistré sur disques - les rubans magnétiques n'existent pas encore - est expédié à douze stations privées du Québec et arrive à destination... en miettes. La Ligue, qui ne peut retenir du temps d'antenne aux stations affiliées au réseau national, doit payer les services des stations privées dont les horaires sont variables et, pour chaque discours, obtenir l'assentiment de censeurs. Tous ces déboires ne font qu'accentuer le sentiment de frustration des représen-

28. AUL, Fonds Adrien-Pouliot, P168/3/1/4/3 «Lettre d'Alphonse de la Rochelle, chef du secrétariat de la Société Saint-Jean-Baptiste de Montréal, à Gladstone Murray, directeur général», 8 avril 1942. Voir également les lettres que de la Rochelle a adressées ce même jour au président de la Ligue pour la défense du Canada et à Adrien Pouliot.

29. ANQ, Fonds SSJB, loc. cit., «Lettre de Henri D'Eschambault, Président de la SSJB du Manitoba, à Alphonse de la Rochelle, chef du secrétariat SSJB, Montréal», 3 mars 1942: 2519. 
tants du NON et les convaincre que la radio nationale n'est qu'un objet d'appropriation du pouvoir politique fédéral ${ }^{30}$.

\section{4 - L'EXACERBATION DES DISCOURS}

La tension monte chez les partisans du NON et les déclarations se font de plus en plus acerbes et politisées. Mais la remarque vaut aussi pour les partisans du OUI. Les journaux canadiens, tels Le Devoir, Saturday Night, la Gazette de Montréal, Winnipeg Free Press, affichent une position officielle et publient parfois des articles ou des éditoriaux sur le plébiscite qui prennent des allures cavalières ${ }^{31}$. Le Devoir dénonce, au nom des principes sacro-saints de la démocratie et de la liberté de parole, le refus aux uns (partisans du NON) et l'acceptation aux autres (partisans du OUI) de se prononcer publiquement sur un «sujet d'extrême gravité». La société d'État est prise à partie et identifiée à une radio totalitaire. On dénonce «la propagande de la radio d'État qui porte dans le même sens [que le OUI]», «la propagande que le gouvernement fait à même les postes de Radio-Canada» ou encore «un organisme d'État usant de procédés relevant du totalitarisme ${ }^{32} \gg$. Certains vont même jusqu'à tenir des propos incendiaires: «De quel droit tout donner à ceux-là, tout refuser à ceux-ci? $\mathrm{Du}$ seul droit du plus fort, droit à la Hitler ${ }^{33}$ ?» André Laurendeau pose directement la question: «Le plébiscite qui s'en vient en est-il un à la Hitler ${ }^{34}$ ?»

Par ailleurs, la situation nourrit la propagande de l'ennemi. Nous présentons ici quelques extraits d'émissions diffusées sur les ondes de la radio allemande à Paris et captées par les services d'ondes courtes de la SRC, émissions qui font référence au plébiscite de 1942:

5 mai 1942 - Le Canada français a refusé de payer un lourd tribut pour une guerre qui n'est pas la sienne;

30. Le plus grand effort de propagande radiophonique du gouvernement fédéral, d'affirmer Renée Legris, est dirigé au Canada vers le Québec, afin de soutenir son idéologie de participation à la guerre. Renée Legris, Propagande de guerre et nationalisme..., 20.

31. Voir les articles suivants: André Laurendeau, «Veut-on miner le prestige de RadioCanada?», Le Devoir, 21 avril 1942; «Nous ne serions plus Canadiens...», Le Devoir, 28 mars 1942. Dans la livraison du 28 mars 1942, le directeur du Devoir, Georges Pelletier, critique de manière acerbe certains articles parus dans Saturday Night et Ottawa Citizen à propos de la question du plébiscite au Québec; «Yes Campaign Gains Montreal», Gazette de Montréal, 11 avril 1942; «The Extremists in Quebec», Winnipeg Free Press, 30 janvier 1942.

32. Ibid. À propos de la propagande que le gouvernement fait à même les postes de RadioCanada, voir Débats, 4 juillet 1942: 4687.

33. Georges Pelletier, «De qui se moquerait-on?», Le Devoir, 24 mars 1942.

34. André Laurendeau, La crise de la conscription..., 107. 
5 juin 1942 - Le Premier ministre est-il dans ses droits lorsqu'il a l'intention d'instaurer la conscription?

3 juin 1942 - Les Canadiens français n'ont rien à retirer de cette guerre $^{35} \ldots$

Comme on le sait, les partisans du NON ont perdu à l'échelle nationale $(36,3 \%)$ lors du plébiscite, mais ils ont gagné largement au Québec $(71,2 \%)^{36}$. Au lendemain du vote, le gouvernement fédéral cherche des moyens pour discréditer la Ligue: «Le bureau de la Ligue pour la défense du Canada devrait faire l'objet d'une surveillance constante; des efforts devraient être faits pour savoir d'où proviennent leurs fonds; [les responsables] des règlements de la défense du Canada devraient exiger la divulgation des sources financières de la Ligue et tout devrait être mis en œuvre pour discréditer les chefs de mouvements subversifs ${ }^{37}$.» $\mathrm{Par}$ ailleurs, à la Chambre des communes, les mêmes arguments reviennent. Le député libéral, Liguori Lacombe, dénonce «[...] les exécrables méthodes hitlériennes qui privent la population de l'exercice d'un droit sacré, celui de connaître la véritée ${ }^{38}$ ». Pour sa part, le député Maxime Raymond s'interroge sur la duplicité du discours véhiculé par le gouvernement King:

Société indépendante? Oui, pour couvrir toute la propagande que le Gouvernement fait à même les postes de Radio-Canada [...]. Or, tous ceux qui étaient en faveur du OUI ont pu parler à la radio [...]. Mais on refusait à tous ceux qui étaient en faveur du NON de se servir du

35. ANC, Fonds Mackenzie-King, 343, «Report On "Paris- Canada" Programme Directed to French Canadians From German Controlled Radio in Paris», September 23, 1942. «Compiled From Broadcasts Picked Up By the Shortwave Monitoring Service of the CBC, Ottawa, Canada», 282134282137. Voir particulièrement la rubrique (c) Plebiscite/Conscription, «Illustrative Quotations». Dès le début des hostilités, l'Allemagne exploite tout discours susceptible de déstabiliser la situation politique au Canada. En 1940, le député de Parkdale, H. A. Bruce, déclare à la Chambre: «Dimanche soir, la radio allemande, au cours d'une émission sur ondes courtes tout particulièrement adressée aux Canadiens français a fait savoir au Québec qu'Hitler lui offre son appui et son entière indépendance. Nous n'avons qu'à nous rebeller contre la Grande-Bretagne et nous l'aurons ensuite notre Laurentie!», Débats, 2 juillet 1940, 1284. Voir également les références suivantes: «The Nazi Radio and Canada», Winnipeg Free Press, 14 mai 1942; ANC, Fonds Brooke-Claxton, «Letter from (?) to Brooke B. Claxton», 2 mai 1942.

36. Voir André, Laurendeau, La crise de la conscription..., 119. Pour une analyse détaillée du vote en faveur du NON, voir: François-Albert Angers, «Un vote de race — analyse mathématique et statistique du vote au plébiscite dans les cinq provinces de l'Est», L'Action nationale, 19 (mai 1942): 299-313. Voir également l'interprétation qu'en donnent Claude Beauregard, Edwidge Munn et Béatrice Richard dans la préface de l'ouvrage de Wilfrid Sanders intitulé Jack et Jacques: l'opinion politique au Canada pendant la Deuxième Guerre mondiale (Montréal, Comeau \& Nadeau, 1996), 9-20.

37. ANC, Fonds Brooke-Claxton, MG32 B5 V. 137, «Suggestion re: Opinion in Canada», article 1d, document non signé et daté du 29 mai 1942 (notre traduction).

38. Débats, 17 juin 1942: 3535. 
réseau national [...]. On parle de liberté, de justice et de démocratie; on devrait commencer à respecter ces principes au Canada avant de nous demander d'aller les faire respecter ailleurs ${ }^{39}$.

On ne peut préciser dans quelle mesure la controverse entre la Ligue et Radio-Canada a influencé les résultats du plébiscite au Québec, mais l'hypothèse que cette affaire a moussé la campagne du NON est plausible. Nous pouvons constater l'impact de cette décision par la diversité et surtout par le degré d'intensité des déclarations, opinions ou commentaires qui ont dérivé de ce refus. Il en est de même pour les allocutions et causeries radiophoniques du NON qui ont été diffusées sur les ondes des stations privées du Québec, notamment CKAC (Montréal) et CHRC (Québec), et qui ont servi de mesure palliative au refus de Radio-Canada pour rejoindre une partie importante des auditeurs québécois regroupés dans ces deux grands centres urbains.

Tous ces discours qui se sont construits ou qui se sont amplifiés tirent leur signification profonde d'une seule et même réalité: le refus de RadioCanada soutenu par l'ingérence politique du gouvernement fédéral. Dès le départ, si la société d'État avait acquiescé à la demande d'André Laurendeau, nous pouvons, de façon générale, émettre l'hypothèse que nombre de discours auraient évolué différemment ou n'auraient jamais existé et, de façon spécifique, que la relation d'opposition «OUI versus NON» se serait articulée autour du contenu, évitant ainsi au discours du NON de dévier de sa trajectoire pour s'attaquer au médium, en l'occurrence RadioCanada, plutôt qu'au message. Inversement, si Radio-Canada avait ouvert ses ondes à la Ligue, n'aurait-elle pas subi des pressions encore plus grandes de la part des tenants du OUI (qui se sont prononcés majoritairement à 63,7\%)? Quoi qu'il en soit, les raisons qui ont motivé le gouvernement fédéral à intervenir dans la décision de la SRC ont modifié considérablement la nature du débat entre le OUI et le NON et surtout ébranlé RadioCanada qui, depuis sa création en 1936, consacre des efforts importants pour asseoir sa crédibilité et démontrer sa neutralité.

On peut aussi voir dans cette controverse et dans cette perte de confiance à l'endroit de Radio-Canada un des motifs centraux du projet de loi sur la radio provinciale (Radio-Québec) que le Premier ministre du Québec, Maurice Duplessis, soumet à l'Assemblée nationale du Québec, en mars 1945. En contrepoint, ce projet n'est pas sans rappeler la frustration de Duplessis au cours de l'élection provinciale de 1939. Ayant refusé de faire approuver son discours radiophonique par le ministre fédéral dont

39. Débats, 4 juillet 1944, 4687. 
relevait la SRC, Duplessis avait été automatiquement exclu des ondes nationales. Mécontent de cette décision, il avait dénoncé l'attitude du gouvernement fédéral lors d'un discours électoral: «M. Lapointe [ministre de la Justice et secrétaire d'État dont relève la SRC] nous attaque, il nous provoque [...]. M. Lapointe et M. Godbout ont laissé les communistes parler à la radio, mais M. Lapointe refuse ce droit au député de TroisRivières, au Procureur général de la province et au Premier ministre du Québec ${ }^{40}$.»

Lors des débats sur le projet de loi d'une radio provinciale, André Laurendeau n'a pas oublié la leçon de 1942. Il maintient que «RadioCanada a été un partisan fanatique de l'impérialisme à travers la province [et que la société d'État] s'est faite le fabricant d'une unité nationale artificielle». On peut prétendre, ajoute-t-il, que la voix du Québec est exprimée sur le réseau français de Radio-Canada, mais c'est une voix singulièrement faussée. La radio nationale, poursuit le chef du Bloc populaire en faisant référence au plébiscite, n'a pas rempli, envers la province de Québec, la mission que l'on était en droit d'attendre d'elle ${ }^{41}$. Laurendeau et d'autres députés de l'Assemblée législative approuvent, avec certaines réserves, le principe de la création de Radio-Québec; ils souhaitent que cette radio serve d'abord les intérêts de la province et qu'elle soit appelée à jouer un rôle de catalyseur de la pensée sociale et politique du Québec ${ }^{42}$.

\section{5 - LA CENSURE ET L'UNITÉ CANADIENNE}

Il nous paraît erroné de limiter, comme le font certains auteurs, l'étude de la censure aux seules interventions qui découlent de l'application de la loi des mesures de guerre ${ }^{43}$. Nous croyons plutôt que sous le couvert de la

40. Maurice Duplessis (discours prononcé par), «Je suis et je serai toujours contre la conscription», Québec, L'Événement- Journal, 4 octobre 1939, 13.

41. Louis Robillard, «Le débat sur le bill de la radio provinciale est engagé», Le Devoir, 14 mars 1945. Voir également Émile Benoist, «Radio-Québec», Le Devoir, 21 mars 1945; «Le plébiscite: M. King et Cardin demandent de faire confiance au gouvernement; M. Duplessis déclare qu'il votera NON», Le Devoir, 25 avril 1942.

42. Dans son article, Louis Robillard fait état des sérieuses réserves émises par Adélard Godbout et André Laurendeau quant à l'éventuelle prise de contrôle du «réseau de postes et de programmes» par un seul homme qui est en même temps le chef du gouvernement et le chef d'un parti. Voir «Le débat sur le bill de la radio provinciale...», loc. cit.

43. Pour une meilleure compréhension de ce propos, voir George D. Kerr, «Skirting the Minefield: Press Censorship, Politics and French Canada, 1940», Canadian Journal of Communication, 8,2 (janvier 1982): 46-64, et particulièrement 48-49; Marc, Raboy, Occasions ratées. Histoire de la politique canadienne de radiodiffusion (Montréal/Sainte-Foy, Liber/Les Presses de l'Université Laval, 1996), 100; ANC, RG2, Série 14, vol. 5968, op. cit., au sous-titre «Suspension of Subversive Publications». 
raison d'État, la censure se manifeste parfois sous des formes plus subtiles, mais non moins présentes. Ainsi, comme on l'a vu, la réponse officielle de la SRC à la Ligue qui ne cesse d'être retardée et le refus qui lui est communiqué à quelques jours seulement du plébiscite ne deviennent-ils pas une forme de censure qu'impose la SRC, et par conséquent le gouvernement fédéral, aux tenants du NON? À cet égard, le témoignage d'Augustin Frigon, directeur général de la SRC, devant le comité spécial de la radiodiffusion (1946) laisse songeur. Lorsqu'un membre du comité (M. Bertrand) lui demande alors pourquoi la SRC a refusé aux tenants du NON de se faire entendre sur les ondes nationales, Frigon répond:

Frigon - Je viens de vous le dire. Je ne sais pas pourquoi. Si vous me demandez mon opinion maintenant, je crois qu'il est possible que ç'ait été une erreur. C'est tout ce que je puis vous dire.

Bertrand - Qui a pu vous demander dans la province de Québec d'exercer ce droit, monsieur Frigon?

Ross $^{44}$ - Je ne crois pas qu'on doive poser cette question.

Ross - Lorsque le projet de loi du plébiscite a été présenté en 1942, c'était une mesure fédérale. Est-ce exact?

$[\ldots]$

Frigon - Oui

Bertrand - Il concernait le Parlement?

Frigon - Oui

$[\ldots]$

Frigon - Je suppose que n'importe lequel individu [en Chambre] avait le droit de parler [à la radio], pourvu qu'il soutienne une bonne cause.

Bertrand - D'après le Livre blanc ${ }^{45}$, vous soutenez que vous étiez en mesure d'accorder du temps à ceux qui partagaient cette opinion?

Frigon - Je crois que nous aurions pu le faire, oui ${ }^{46}$.

Par ailleurs, dès 1943, certains journalistes du Canada anglais reprochent au gouvernement fédéral d'avoir exercé une ingérence politique à l'endroit de Radio-Canada et ils accusent la société d'État d'avoir muselé la Ligue et les

44. Un autre membre du comité.

45. Voir la note infrapaginale $\mathrm{n}^{\mathrm{O}} 11$.

46. Canada, Comité spécial de la radiodiffusion, 1946, Procès-verbaux et témoignages, Fascicule $\mathrm{n}^{\mathrm{o}} 11$ (Ottawa, Edmond Cloutier, 6 août 1946), 8-9. 
représentants du NON. Grant Dexter du Winnipeg Free Press se demande si on peut parler «d'indépendance de la SRC ou si nous avons une radio nationale qui produit des émissions qui plaisent à ceux qui détiennent le pouvoir politique au Canada ${ }^{47}$ ». En contrepartie, le seul blâme que les journalistes se disent prêts à accepter, c'est de n'avoir pas réagi au moment où la société d'État a refusé les ondes aux tenants du NON. B. K. Sandwell, rédacteur en chef du Saturday Night, explique: «cette politique [de la SRC] était non seulement illogique; elle était malavisée [...]. Nous devons aujourd'hui nous frapper la poitrine pour avoir omis d'élever la voix contre cette mesure malavisée ${ }^{48}$.» Une telle déclaration ne laisse pas indifférent, a fortiori lorsque nous constatons que, sur d'autres questions concernant le plébiscite, Sandwell et Laurendeau avaient des opinions différentes et bien arrêtées ${ }^{49}$.

D'un autre côté, nous pouvons nous interroger sur les propos de Raboy qui affirme que «les critiques communistes de la politique de guerre étaient réprimées plus durement que ne l'étaient les nationalistes canadiensfrançais ${ }^{50} \gg$. Que dire, par exemple, du comité parlementaire de 1942, chargé d'étudier les règlements de l'organisme fédéral responsable de la défense du Canada et qui a recommandé, par un vote majoritaire, de lever l'interdit contre le parti communiste du Canada? Le journal L'Action catholique commente l'appui du comité en ces termes: «La campagne du plébiscite aura eu, entre autres résultats, de permettre au Parti communiste canadien une nouvelle apparition sur la scène publique, et cela en dépit du fait que le communisme est illégal en notre pays [...]. Au cœur même de la capitale canadienne on a publié le manifeste de Tim Buck, le chef communiste interné ${ }^{51}$.» L'Action catholique écrit encore: «Une forte pression se fait à Ottawa pour que Tim Buck et les autres chefs communistes puissent reprendre leur activité condamnée comme subversive depuis l'entrée du Canada en guerre. Maintenant que la Russie est devenue notre alliée, le Parti communiste encourage l'effort de guerre auquel il était autrefois opposé et demande en conséquence d'être reconnu comme un groupement légal ${ }^{52} . »$ Étonnamment,

47. Grant Dexter, «Free Discussion on the CBC», Winnipeg Free Press, 16 novembre 1943. Voir également sur cette même page l'article intitulé «The Citizens' Forum».

48. «Of Old Errors», Saturday Night, 29 janvier 1944.

49. Voir, par exemple, CRLG, Fonds André-Laurendeau, P2/F, 111, «La conscription et le prétendu droit de veto du Québec», texte dactylographié et annoté (sd).

50. Marc Raboy, Occasions ratées..., 100.

51. «Sommes-nous des aveugles?», L'Action catholique, 25 mars 1942.

52. «Tim Buck et la révolution», L'Action catholique, 6 juillet 1942. Voir également les articles de journaux suivants: Charles Gauthier, «La réhabilitation du Parti communiste», Le Droit, 23 juillet 1942; Gustave Savard, «Le communisme vis-à-vis de la loi», Le Droit, 25 juillet 1942; LouisPhilippe Roy, «Un parti qui veut la révolution», L'Action catholique, 27 juillet 1942; Louis-Philippe Roy, «Veut-on légaliser la trahison?», L'Action catholique, 28 juillet 1942. 
Radio-Canada accorde, quatre ans plus tard, «à Tim Buck, chef OuvrierProgressiste-Communiste, la faveur d'adresser la parole sur le réseau d'État, en faveur de son parti». Dans une lettre de protestation que fait parvenir Stanislas Vachon, secrétaire de la Ligue des Patriotes, à Augustin Frigon nous pouvons lire: «Il est inconcevable, après l'enquête sur l'espionnage russe et les graves accusations qu'elle contient contre le parti ouvrier-progressiste, dont le seul député à Ottawa purge actuellement une sentence pour trahison, qu'on permette à son chef de faire de la propagande publique, avec l'argent des contribuables, en faveur d'un mouvement reconnu et condamné comme subversif ${ }^{53} . »$

Radio-Canada se serait-elle libéralisée ou aurait-elle tiré une leçon quelconque du plébiscite au point de ne plus bâillonner les représentants de partis, groupes ou organismes à tendance subversive? Nous avons affirmé, en début d'analyse, que la propagande subversive ne constitue pas, au cours de la Deuxième Guerre mondiale, une menace véritable pour le Canada. Par contre, l'unité canadienne qui devient, particulièrement au cours de cette guerre, la pierre angulaire de la politique du gouvernement King doit être préservée à tout prix ${ }^{54}$. Comme le rappelle l'historien J. L. Granatstein, «King croyait sincèrement que seul le parti libéral pouvait sauvegarder l'unité canadienne pendant toute la guerre ${ }^{55}$. Le Bloc populaire qui est né de la Ligue pour la défense du Canada et qui, sur la scène provinciale, a pour chef, André Laurendeau, subit inexorablement le contrecoup des manœuvres politiques fédérales exercées à l'endroit des partisans du NON. Les propos de Blair Fraser, journaliste au MacLeans, font voir, encore une fois, le ressentiment du Bloc qui s'inscrit dans le sillage de la Ligue: «le Bloc populaire [...] n'a jamais oublié que, lors de la campagne du plébiscite, la SRC ait refusé du temps d'antenne aux partisans du NON. [...] En ce qui a trait à la radiodiffusion de sujets à caractère politique, le Bloc estime que les nouvelles règles de la SRC ont été faites sur mesure pour l'empêcher de se faire entendre sur les ondes [de la société d'État] ${ }^{56}$.» Ce constat ne peut que nous éclairer sur la réponse que donne Maxime Raymond, ancien membre de la Ligue pour

53. CRLG, Fonds Maxime-Raymond, P3/ F, 366, «Lettre de Stanislas Vachon à Augustin Frigon», 24 août 1946.

54. George D. Kerr, «Skirting the Minefield...», 46, affirme que la censure de la presse demeure une question controversée non pas pour la cause elle-même, mais plutôt parce qu'elle remet à l'ordre du jour la question la plus déchirante du pays, à savoir la différence de perception sur l'utilité et les buts de cette guerre que les deux peuples fondateurs entretiennent.

55. J. L. Granatstein, «Le Québec et le plébiscite de 1942 sur la conscription», Revue d'histoire de l'Amérique française, 27,1 (juin 1973): 43-62, et particulièrement 44.

56. Blair Fraser, «Static on the CBC», MacLean's, $1^{\text {er }}$ juin 1944, 16, 54 (notre traduction). 
la défense du Canada et nouvellement député fédéral du Bloc (Beauharnois-Laprairie), à Stanislas Vachon, secrétaire de la Ligue des Patriotes: «Vous m'avez demandé si on accordait des émissions gratuites au Bloc populaire canadien. Je vous réponds que non, pas plus qu'on nous a permis lors du plébiscite de la conscription, de parler en faveur du non ${ }^{57}$.»

L'assouplissement des règles en faveur du parti communiste au Canada et le maintien de la ligne dure envers le Bloc populaire, pendant et après la guerre, traduisent bien la continuité des intentions politiques fédérales, à savoir d'harmoniser ses relations avec ceux qui partagent, à l'échelle internationale, des intérêts politiques communs et définis par les pays alliés, d'une part, et d'écarter, à l'échelle nationale, tout discours qui pourrait nuire à l'unité nationale, d'autre part.

\section{6 - LES ADMINISTRATEURS DE LA SOCIÉTÉ RADIO-CANADA}

C'est, bien sûr, en relation avec la question nationale que la décision controversée de Radio-Canada lors du plébiscite aura des conséquences négatives sur la société d'État. Les partisans du NON écartés des ondes provenaient, en majorité, des francophones du pays. Les effets de la politique suivie par Radio-Canada lui porteront un tort considérable, en plus d'ébranler la confiance de ses administrateurs.

On comprend aisément que le rôle d'Augustin Frigon, comme principal représentant et porte-parole de la SRC, prête le flanc à la critique. La tiédeur de son engagement dans cette affaire le place dans une situation pour le moins vulnérable. Le fait que la Ligue s'adresse à Frigon n'est pas sans fondement. Pour faire mieux saisir la portée de notre propos, nous devons remonter à la fin des années 1920 lorsque la Commission royale d'enquête sur la radiodiffusion (nommée commission Aird) a pour mandat d'enquêter sur les conditions de la radiodiffusion au Canada. Seul ingénieur parmi les trois membres de cette commission, Augustin Frigon demeure, en grande partie, responsable des études techniques reliées à cette enquête. L'auteure, Mary Vipond, affirme que «Frigon fut très utile pour expliquer les aspects techniques aux autres commissaires; de plus, et cela est inévitable, sa principale préoccupation fut la protection des intérêts culturels spécifiques au Canada français (et particulièrement, ceux de la province de Québec) dans la formulation d'une politique nationale de la radio ${ }^{58}$.»

57. CRLG, Fonds Maxime-Raymond, loc. cit., 366, «Lettre de Maxime Raymond à Stanislas Vachon», 27 août 1946.

58. Mary Vipond, Listening In: the First Decade of Canadian Broadcasting, 1922-1932 (Montréal/Kingston, McGill-Queen's University Press, 1992), 211. 
Lors de la création de la SRC, en 1936, Frigon est nommé directeur général adjoint. À l'instar de Leonard W. Brockington, président de la SRC, et de Gladstone Murray, directeur général, sa nomination ne suscite que des éloges et beaucoup d'optimisme. Sa réputation d'homme intègre, et surtout ses compétences en matière de radiodiffusion comme ingénieur et comme membre de la commission Aird, lui valent la confiance des gens du milieu. Cette reconnaissance est d'ailleurs officiellement soulignée par les membres du Bureau des gouverneurs de la SRC qui, en 1940, lui réaffirment leur confiance, tout en lui témoignant ainsi qu'à Gladstone Murray de leur gratitude pour services rendus au cours des quatre premières années d'existence de la SRC: «Notre radio nationale, sous leur direction, a permis d'en arriver à un service public de grand calibre et, au cours de cette période de guerre, avec tout ce que cela comporte de diversion et de bouleversements, cette radio a été un facteur puissant pour la promotion de l'unité nationale au pays de même qu'un facteur de compréhension et de bienveillance à l'étranger ${ }^{59}$.»

En s'adressant officiellement à Augustin Frigon plutôt qu'à Gladstone Murray, André Laurendeau fait non seulement appel au directeur général adjoint, mais également à la personne responsable du réseau français au Canada. En poste à Montréal, Frigon demeure conscient des tensions que soulève le plébiscite dans cette ville et il doit agir avec circonspection s'il ne veut pas s'aliéner la sympathie de la population francophone du Québec. À cet égard, il devra supporter pendant longtemps les effets de cette délicate situation. Ironie du sort, c'est au Canada anglais que Frigon doit essuyer les pires reproches. En 1943, lors d'une réunion du Bureau des gouverneurs, la majorité des membres font savoir à leur président qu'ils ne sauraient accepter la nomination de Frigon à titre de directeur général de la SRC. Malgré la loyauté et l'appui incontestable dont il a fait preuve envers la société d'État lors du plébiscite de 1942, Frigon ne peut inspirer une confiance totale aux gouverneurs. Ces derniers justifient notamment leur position en soutenant que:

Dr Frigon a été responsable à part entière du réseau canadienfrançais et par sa très grande spécialisation dans ce domaine, il ne

59. ANC, Fonds Board of Governors CBC/SRC, RG41, 722, 2, «CBC - Resolutions passed by Board of Governors. Resolution: Assistant General Manager, confidence in. Minutes of the $16^{\text {th }}$ meeting of the Board of Governors of the Canadian Broadcasting Corporation held in Ottawa, November 26, 1940», 291. 
peut être sensible aux demandes et aux besoins des autres parties du Canada;

$[\ldots]$

En cette période pour le moins particulière, des sentiments raciaux se sont manifestés au Canada et, selon l'opinion de la majorité du bureau, la nomination d'un Canadien français, à titre de directeur général, pourrait stimuler dangereusement le sentiment anti-français qui s'est développé ${ }^{60}$.

Ces raisons contrastent avec les éloges que ces mêmes gouverneurs lui adressaient à l'unanimité, en 1940. Même si la nomination de Frigon reste étroitement liée au «fait français» — pour reprendre l'expression de Frank W. Peers - il n'en demeure pas moins que le plébiscite de 1942 a eu pour effet de miner, au plus haut niveau décisionnel de la société d'État, le climat de confiance qui régnait à son égard. Ce désaveu de la part des gouverneurs n'a finalement pas fait obstacle à sa nomination de directeur général, mais toute cette mauvaise presse fera de lui un administrateur vulnérable qui sera sévèrement critiqué dès qu'il tergiversera ou qu'il prendra une décision dont l'équité sera susceptible d'être mise en doute $^{61}$.

\section{7- LE PLÉBISCITE DE 1942 ET LE RÉSEAU FRANÇAIS DE RADIO- CANADA}

Malgré les déboires de certains de ses administrateurs, Radio-Canada connaît, en cette période de conflit mondial, un essor sans précédent de sa programmation et de son développement technique. L'épisode du plébiscite a-t-il eu un impact sur le développement du réseau français de Radio-Canada?

Les recherches historiques sur l'évolution du réseau français de RadioCanada sont peu nombreuses. La plupart des historiens canadiens se limitent, pour l'essentiel, à faire l'apologie d'un seul et unique système public, englobant ainsi le «fait français» dans l'évolution de ce système ou considérant ce fait comme une sorte d'appendice du réseau national. À l'exception de quelques auteurs, tels E. Austin Weir, Frank W. Peers, Laurier Gareau, Elzéar Lavoie ou Rossel Vien qui ont su présenter une étude plus rigoureuse de la radio d'expression française nationale, l'analyse historique s'intéresse davantage aux forces politiques et économiques qui ont influencé le développe-

60. ANC, Fonds Mackenzie-King, 345, «New Appointments», 296981.

61. Voir Débats, 25 février 1944, 912; Blair Fraser, «Political Suppression», Gazette de Montréal, 27 février 1945; Blair Fraser, «Of Old Errors», Saturday Night, 29 janvier 1944. 
ment de ce réseau qu'à la détermination des Canadiens français qui ont lutté solidairement pour l'obtention de stations à l'extérieur du Québec ${ }^{62}$. De ce point de vue, nous ne pouvons admettre le lien de causalité implicite dans cette affirmation de Greg Marc Nielsen - qui reprend les propos de Marc Raboy: «L'expansion rapide du système technique au Québec et au Canada français coïncide avec le fait que l'État devait absolument améliorer ses relations avec le Québec après la crise de la conscription de $1942^{63}$.» Une telle approche ne saurait satisfaire aux exigences de notre analyse qui s'appuie sur des considérations différentes.

L'effet de la guerre sur le progrès technique demeure une question controversée: en imposant ses priorités, la guerre rompt le rythme de croissance normal d'une société, mais il n'en demeure pas moins qu'elle accélère le progrès scientifique et technique. Il n'est pas de notre intention de débattre de cette question, mais plutôt de poser, à partir de ce constat, un regard scrutateur sur le développement technique de la SRC pendant la Seconde Guerre mondiale.

En 1939, la société d'État procède à la mise en place de la deuxième étape de son plan de rayonnement et, en 1941, pas moins de neuf émetteurs dont quatre de très grande puissance $(50 \mathrm{~kW})$ desservent les grands centres urbains et leurs régions périphériques. Entre 1940 et 1945, la SRC inaugure son service national de nouvelles, crée un service de radiodiffusion international sur ondes courtes et met en service cinq stations en modulation de fréquence (MF), sans compter les services techniques et projets spécifiques liés aux besoins de la guerre (service d'émissions bilatéral avec la Grande-Bretagne, cars blindés pour les reportages du front, collaboration avec les Forces françaises libres pour l'établissement d'une station à ondes courtes en Afrique équatoriale française, etc.). En 1944, un deuxième réseau anglais, appelé Dominion, s'ajoute au réseau Trans-Canada et relie 34 stations privées d'un bout à l'autre du pays. Immédiatement après la guerre, Radio-Canada procède à la troisième étape de son plan de rayonnement national en ajoutant à son réseau deux autres émetteurs puissants (Alberta et Manitoba), en construisant des émetteurs-relais en Colombie-Britannique et en Ontario et en mettant en œuvre ses projets les plus urgents d'immobilisation grâce à un prêt

62. Frank Peers, The Politics of Canadian Broadcasting, 1920-1950 (Toronto, University of Toronto Press, 1969); E. Austin Weir, The Struggle for National Broadcasting in Canada (Toronto, McClelland \& Stewart Ltd., 1965); Laurier Gareau, Le défi de la radio française en Saskatchewan (Régina, Société historique de la Saskatchewan, 1990); Elzéar Lavoie, «L'évolution de la radio au Canada français avant 1940», Recherches sociographiques, 12,1 (janvier-avril 1971): 17-50; Rossel Vien, Radio française dans l'Ouest (Montréal, Éditions Hurtubise HMH, coll. «Communications», 1977).

63. Greg Marc Nielsen, Le Canada de Radio-Canada, sociologie critique et dialogisme culturel (Toronto, Éditions du Gref, coll. «Theoria», $\mathrm{n}^{0} 4$, 1994), 83; Marc Raboy, Opportunités ratées..., 98-99. 
consenti par l'État. Bref, les projets d'expansion nationaux et internationaux se poursuivent à un rythme accéléré, mais les retombées pour le réseau français restent bien minces en comparaison des deux réseaux anglais existants. Le rapport de la Commission royale d'enquête sur la radio et la télévision (rapport Fowler) fait d'ailleurs état de cette situation déplorable en affirmant: «[qu'] avec les chiffres du recensement de 1951 à l'appui, [...] plusieurs groupes de langue française, numériquement importants et situés dans diverses parties du Canada, ne jouissent d'aucun service de diffusion française, ou reçoivent un service si restreint dans le temps et si pauvre en qualité, que cela revient à aucun service du tout ${ }^{64}$.»

La solidarité des communautés francophones du pays et les actions concertées de leurs porte-parole, de même que l'appui inconditionnel de Canadiens français qui occupent des postes clés au sein d'organismes publics, religieux ou privés constituent, à notre avis, les véritables assises du réseau d'expression française au pays ${ }^{65}$. Si l'on avait voulu favoriser l'expansion rapide du système technique au Québec et au Canada français, afin d'améliorer les relations entre le gouvernement fédéral et le Québec, comme le laisse entendre Greg Marc Nielsen, comment expliquer qu'Adrien Pouliot, membre du Bureau des gouverneurs, réussisse à «arracher un compromis» de ses collègues anglophones du Bureau pour l'octroi d'un permis d'une station d'expression française dans les Prairies alors que la demande originale des requérants fait état de quatre stations ${ }^{66}$ ? La résolution prise par le Bureau des gouverneurs, le 8 mai 1944, indique que ces derniers sont prêts à approuver

64. Robert Fowler, Rapport de la Commission royale d'enquête sur la radio et la télévision (ciaprès Rapport Fowler) (Ottawa, Imprimeur de la Reine, 1957), 269; voir également Vincent Massey, Rapport de la Commission royale d'enquête sur l'avancement des arts, lettres et sciences au Canada, 1949-1951 (ci-après Rapport Massey) (Ottawa, Edmond Cloutier, 1951), 44, article 43.

65. À cet égard, le rapport Fowler (p. 273) est on ne peut plus explicite: «les quatre [stations françaises des Prairies] ont pu être lancées grâce à des dons recueillis dans les trois provinces des Prairies et dans la province de Québec [...]. Leur existence même est une œuvre de dévouement continu et de sacrifices quotidiens de la part de la direction et du personnel». Voir également abbé Antoine D'Eschambault, «Radio-Saint-Boniface», Vie française, 1,1 (1946-1947): 163-69; Charles Papen, Radio-Prairie-Nord Limitée, mémoire présenté par Radio-Prairie-Nord Limitée à la Commission Fowler sur la question de la télévision et la radiodiffusion au Canada (sd).

66. La correspondance de divers porte-parole et représentants des communautés francophones du pays (Adrien Pouliot, abbé Maurice Baudoux, abbé Paul-Émile Gosselin, Raymond Denis, etc.) témoigne en faveur de notre propos. À cet égard, voir AUL, Fonds Adrien-Pouliot, P168/4/1/5 «Lettre d'Adrien Pouliot au Dr L.-O. Beauchemin», 5 janvier 1944 et «Lettre du Cardinal Rodrigue Villeneuve à Adrien Pouliot», 11 janvier 1945; AUL, Fonds Adrien-Pouliot, P168/3/2, «Lettre de Maurice Baudoux à Adrien Pouliot», 2 juin 1944; ANQ, Fonds SSJB, 06-M, P82/35-183 document microfilmé 7987, «Lettre de J.Léopold Gagnon à l'Association canadienne-française de l'Alberta, 14 juin 1949, 1198, 1200 et «Lettre de L.-O. Beauchemin à la SSJB de Montréal», 15 juin 1949; ANC, Fonds Board of Governors CBC/SRC, RG41, 752; «Minutes of the 36th. Meeting of the Board of Governors of the CBC Held in Montebello, P.Q., May 7th. and 8th. 1944», 597. 
la demande d'une seule station d'expression française (Saint-Boniface, Manitoba), «attendu qu'il n'en coûte rien à la SRC pour l'érection, l'équipement, l'opération et l'entretien de la dite station ${ }^{67} \gg$. Comment justifier la levée de fonds que le Québec organise conjointement avec les provinces des Prairies pour financer, en 1946, la construction de la première station d'expression française dans cette région canadienne? Nous pourrions ainsi cheminer longuement en multipliant les exemples d'appui, d'entraide et de solidarité des Canadiens français pour l'obtention de stations à travers le pays.

Ainsi, à la suite de ces rappels historiques, nous pouvons affirmer que la crise de la conscription de 1942 n'a jamais joué le rôle de catalyseur de l'expansion du système technique de radiodiffusion au Québec et au Canada français. Si la crise a accentué l'expansion d'un système technique, c'est bien celui du réseau anglais! Alors que les provinces des Maritimes possèdent leur première station d'expression française en 1953, c'est-à-dire 11 ans après le plébiscite, un second réseau d'expression anglaise (Dominion) voit le jour en $1944^{68}$. Ce réseau, soutient la SRC, est «destiné à permettre un deuxième choix d'émissions». Que dire alors du réseau d'expression française qui, en 1944, en est encore à ses premiers balbutiements?

\section{CONCLUSION}

La fermeture des ondes aux partisans du NON ne fait que s'ajouter aux difficultés que rencontre la SRC dans la mise en œuvre de son mandat. L'année 1942 est également marquée d'une série d'événements qui remettent en question l'autorité et l'efficacité de la société: le directeur général, Gladstone Murray, est limogé après que le Comité spécial de la radiodiffusion (1942) eut adressé de sévères réprimandes à la direction et au Bureau des gouverneurs. Au cours de cette même année, son successeur, James $\mathrm{S}$. Thomson, est contraint de démissionner après avoir refusé de diffuser le discours du chef sortant du Parti conservateur, Arthur Meighen, prononcé lors d'un important congrès à la direction du parti69 .

67. ANC, Fonds Board of Governors, 601. Pour un résumé de la discussion entre les membres du Bureau, voir Archives de la Saskatchewan, Document R-128, «Lettre de Raymond Denis à l'abbé Maurice Baudoux», 11 mai 1944.

68. Pour un aperçu de ce contexte: Université de Moncton, Archives du Centre d'études acadiennes, Fonds Clément-Cormier, 177.620, «Lettre de Léopold Houlé au père Clément Cormier», 24 mai 1952; André Laurendeau, Radio, télévision et vie française (Montréal, Éditions Bellarmin, «Institut social populaire», $\mathrm{n}^{\circ}$ 485, juillet-août 1956. 3 et ss.; Rapport Massey, op. cit., 346, article 71, «Rayonnement et émissions des postes de langue française».

69. Dans une lettre adressée au député J. L. Ilsley, Brooke Brian Claxton écrit: «Face au gâchis de la SRC, la solution [de rechange] Thomson-Murray n'augure pas très bien pour l'avenir», ANC, Fonds Brooke-Claxton, «Suggestions re Opinion in Canada», 29 mai 1942. 
L'Association des radiodiffuseurs canadiens, qui témoigne pour la première fois devant le Comité spécial de la radiodiffusion (1942), marque la société d'État du stigmate de «juge et partie» et prépare ainsi la voie à un débat qui ne fera que s'amplifier au cours de la décennie ${ }^{70}$. Force est de constater que la SRC ne dispose ni de la force ni de l'équilibre nécessaires au sein de son organisation pour asseoir sa crédibilité et créer un climat de confiance vis-à-vis de la population. À court et à moyen termes, elle aura du mal à rectifier le tir. Le 25 février 1944, le député, John G. Diefenbaker, déclare devant la Chambre:

L'un des plus graves dangers qui menacent cette Société est la possibilité qu'elle devienne un ballon politique. Plusieurs redoutent que l'absence de directives ces derniers mois ne viennent confirmer leurs craintes sur ce point. Le Bureau actuel des gouverneurs a perdu, semble-t-il, la confiance de la population ${ }^{71} \ldots$

Parce qu'elle n'a pas su faire preuve d'une plus grande autonomie politique lors du plébiscite de 1942, la SRC devient inexorablement «associée à l'appareil répressif de l'État et [...] la radiodiffusion "publique" se valut, pour la première fois également, le vocable de la radiodiffusion "d'État"72». Dans une lettre adressée à Augustin Frigon, André Laurendeau écrit: «Ce n'est pas la Société Radio-Canada qui prend la décision finale, mais bien le gouvernement canadien. Par un coup de force plus digne des pays totalitaires que d'un État démocratique, le ministère s'empare donc de la radio officielle du pays ${ }^{73}$.»

Cette nouvelle étiquette, à forte connotation négative, risque fort de ternir l'image de la société d'État, a fortiori lorsqu'elle s'inscrit dans le contexte de la Deuxième Guerre mondiale et de ses nombreuses références aux pays totalitaires! En cette année de plébiscite, les dénonciations répétées de la Ligue, de représentants d'organismes provinciaux et nationaux, de députés et de journalistes à l'endroit de la SRC, l'hostilité du Parti conservateur qui identifie la société d'État au gouvernement King et l'instabilité au niveau de la direction de ladite société viennent s'ajouter au sentiment d'injustice qui habite la population francophone du pays face à l'attitude de Radio-Canada à l'endroit des tenants du NON. Selon nous, les administrateurs de Radio-Canada n'ont pas pleinement mesuré les conséquences de ce refus et, encore moins, l'ingérence politique qui le

70. Voir Rapport Massey, 330; Débats, 31 mars 1950, 1512.

71. Débats, 25 février 1944, 908.

72. Marc Raboy, Opportunités ratées..., 102-103.

73. CRLG, Fonds Maxime-Raymond, loc. cit., 366 «Lettre d'André Laurendeau à Augustin Frigon», 9 avril 1942 (c'est Laurendeau qui souligne). 
conditionne: le témoignage d'Augustin Frigon devant le Comité spécial de la radiodiffusion, en 1946, le montre bien.

Cet épisode de l'histoire de la radio publique au Canada rappelle, une fois de plus, la nécessité pour les administrateurs de la SRC d'appuyer, avec toute la rigueur et l'impartialité nécessaires, leurs jugements et leurs décisions sur les principes fondamentaux du mandat qui leur a été confié et ce, dans le plus grand respect de la pluralité des opinions. Réparer les erreurs commises et redevenir maître des événements constituent une $œ u v r e$ de longue haleine. À cet égard, les propos de Raymond David, vice-président et directeur général de la radiodiffusion française de Radio-Canada, devant les membres de l'Institut d'administration publique du Canada réunis à Québec, le 27 mars 1979, témoignent d'un enseignement des erreurs du passé:

Il n'est guère possible à une radiotélévision d'être le ferment démocratique et culturel d'une société si elle ne jouit pas d'une indépendance garantie à l'égard des pouvoirs [...]. Nous nous sommes efforcés de traiter de la façon la plus approfondie possible les dossiers présentés au public, parce que nous avons la conviction qu'une information partielle risque souvent d'être partiale ${ }^{74}$.

Le droit pour l'ensemble des citoyens du Canada de faire valoir leur point de vue sur le plébiscite était primordial autant pour la liberté d'expression que pour le maintien d'une saine démocratie institutionnelle. Le rapport de la Commission internationale d'étude des problèmes de la communication (1980) est on ne peut plus explicite sur ces questions: «Quand les voix des dissidents sont réduites au silence, c'est la crédibilité des organes d'information qui est en jeu. En imposant le silence à la controverse, l'État donne à penser qu'il la redoute et qu'il n'est pas aussi assuré de sa vérité qu'il le prétend ${ }^{75} \ldots$..»

74. Raymond David, Radio-Canada et la culture québécoise (Ottawa, Service des relations publiques, 1979), 6 .

75. Sean MacBride, dir., Voix multiples un seul monde (Dakar/Paris, Les nouvelles éditions africaines/La documentation française, UNESCO, 1980), 25. 